\title{
Determination of the Gravitational Potential of Seyfert Cores from High-Resolution Spatial and Spectral Data
}

\author{
M. Wittkowski ${ }^{1}$ \\ Max-Planck-Institut für Radioastronomie, Auf dem Hügel 69, D-53121 \\ Bonn, Germany
}

\begin{abstract}
We summarize the current state of our project to determine the gravitational potential in the innermost few hundred parsecs of Seyfert cores from observed line-of-sight velocities of molecular clouds. Therefore we distinguish between the observational part on the one hand and the theoretical aspects on the other. On the theoretical side the problem is lack of knowledge of the flow pattern and the spatial distribution of the clouds. We analyze the possibility of distinguishing between the different flow patterns and determining the mass distribution. On the observational side the problem is obtaining sufficiently high spatial and spectral resolution.
\end{abstract}

\section{Theoretical Aspects}

We invert the problem of determining the unprojected positions of clouds from the known potential and measured line-of-sight velocities in our Galactic center, which was solved by von Linden, Duschl, \& Biermann (1993), Biermann, Duschl, $\&$ von Linden (1993), and von Linden et al. (1993b). For external galaxies, we have in most cases the advantage of not being located in the galactic plane. In addition to the two positional coordinates, we can also observe the line-of-sight (LOS) velocities. The problem is that we do not really know the flow pattern of the molecular clouds, e.g., whether it is purely circular motion or not, or about the 3-D spatial distribution of the observed clouds in general. So we investigate first to what extent one can determine the flow pattern and the distribution of the clouds. Then we investigate whether or not and to which accuracy it is possible to determine the gravitational potential or the mass parameters respectively. Therefore we assume a two-component model for the mass distribution consisting of a central mass and a background distribution. As far as motion is concerned, we distinguish between general Keplerian orbits, purely circular motion and circular motion superimposed by a radial accretion flow. With these physical models we describe closed orbits, accretion disks, and inflow and outflow. We calculate by means of a Monte-Carlo technique the observable distribution of LOS velocities for the respective gravitational potential. Then we decide by comparison to what extent it is possible to distinguish between the different

\footnotetext{
${ }^{1}$ Also affiliated with Institut für Theoretische Astrophysik, Tiergartenstraße 15, D-69121 Heidelberg, Germany.
} 
flow patterns and the different mass-parameters. As one example we have taken a central mass of $M_{\mathrm{c}}=10^{8} M_{\odot}$ and a background distribution as determined for our Galactic center. We found that it is possible to distinguish between the different flow patterns. To determine the central mass, one has to observe clouds near the center $(r<20 \mathrm{pc})$; to determine the background distribution only the clouds far off the center are convenient. For a detailed description and results with accuracies see Wittkowski \& Duschl (1996).

\section{Observations}

In order to resolve particular molecular clouds within the central part of Seyfert nuclei, high-resolution techniques are needed. We use the Speckle-Masking method (Weigelt 1977; Lohmann, Weigelt, \& Wirnitzer 1983) in the optical and near-infrared wavelength range. In the optical range, cores of several Seyfert nuclei have been resolved in the past using this method. See, for example, Hofmann, Mauder, \& Weigelt (1989), and Mauder et al. (1992).

As a first example, in the near-infrared range, we observed the Seyfert 1 galaxy NGC 4151 with the SAO 6-m telescope on 1996 April 4 through a $K$ band filter $(2191 \mathrm{~nm} / 411 \mathrm{~nm})$. The image of the galaxy shows no evidence of any extended structure within the reached resolution of 130 mas and the dynamic range down to $3 \%$ of the maximum. Comparison with the transfer function shows that the upper limit for the size of the core is 20 mas.

In order to obtain the LOS velocities of the clouds we have built an emissionline objective-prism spectrograph for the $\mathrm{Br} \gamma-$ and the $\mathrm{H}_{2}(1-0)-$ line with a resolution $\lambda / \delta \lambda=8600$. This instrument will enable us to determine LOS velocities with an accuracy of $35 \mathrm{~km} \mathrm{~s}^{-1}$.

Acknowledgments. The results regarding NGC 4151 are based on observations collected at the SAO 6-m telescope in Russia. We thank Y. Balega and everyone involved in the observations for help during the observations, and W.J. Duschl and G. Weigelt for helpful discussions.

\section{References}

Biermann, P. L., Duschl, W.J., \& von Linden, S. 1993, A\&A, 275, 153.

Hofmann, K.-H., Mauder, W., \& Weigelt, G. 1989, in Extranuclear Activity in Galaxies, ed. E. J. A. Meurs \& R. A. E. Fosbury.

Lohmann, A. W., Weigelt, G., \& Wirnitzer, B. 1983, Appl. Optics, 22, 4028.

Mauder, W., Appenzeller, I., Hofmann, K.-H., Wagner, S.J., Weigelt, G., \& Zeidler, P. 1992, A\&A, 264, L9.

von Linden, S., Duschl, W. J., \& Biermann, P. L. 1993, A\&A, 280, 468.

von Linden, S., Biermann, P.L., Duschl, W.J., Lesch, H., \& Schmutzler T. 1993b, A\&A, 280, 468.

Weigelt. G. 1977, Optics Comm., 21, 55.

Wittkowski. M., \& Duschl, W. J. 1996, submitted to A\&A. 